\section{COMPARISON OF TAGUCHI'S PARTIAL CORRECTION TECHNIQUE WITH CONTROL CHARTS}

LUBOS KOTEK $^{1}$, ALES NOSEK ${ }^{2}$, ALOIS FIALA ${ }^{1}$, JIRI ZAHALKA ${ }^{1}$

${ }^{1}$ Brno University of Technology, Faculty of Mechanical Engineering, Institute of Production Machines, Systems and Robotics, Department of Quality, Reliability and

Safety, Brno, Czech Republic

2Brno University of Technology, Quality Management Department, Brno, Czech Republic

DOI: 10.17973/MMSJ.2015_10_201539

e-mail: kotek.l@fme.vutbr.cz

This article compares the most commonly used methods of statistical quality control (control charts - in our case the control chart of individual values) with the Taguchi's method of Partial Correction. The comparison applies to an actual process of hole reaming, and a test is carried out of gradual change of setting (continuously increasing simulation deviation).

KEYWORDS

statistical process control, control charts, Taguchi's partial correction, hole reaming, Beta correction

\section{INTRODUCTION}

Statistical Process Control (SPC) uses statistical methods for preventative quality control, which consists in the implementation of process interventions in the event of significant deviations from predefined levels for its control. The aim of this control is to make the process exhibit a long-term stability of the expected values. [Atienza 1997]

Generally, each process has its own variability, which is a natural component of the process concerned. In practice this means that it is not possible to produce two perfectly identical products; however by controlling this variability can achieve a range within the defined limits. From this it follows that the variability may be of two kinds; the variability arising from random causes and the variability arising from definable / identifiable causes. The variability from random causes can be described as a large number of random and hardly identifiable causes that insignificantly contribute to the overall process variability. If there is only this variability, we are talking about a statistically steady state of the process, the process that is reproducible. Variability that arises from definable causes is the variability that does not act during a standard implementation of the process. Its effect is such that it gives rise to variations that cause irreproducibility of process quality factors. Definable causes of variability occur either suddenly and last for a short time, or for a certain time causing more permanent fluctuations. The methods of statistical process control allow the users both to identify a statistically unstable state, and to intervene into the process, which offsets the effect of a definable cause. Then, in practice, a production facility is not adjusted after the manufacture of products beyond the tolerance range, but the intervention into the process is carried out at a time when there is already a high probability that in the near future (calculated by time or number of units produced) specifications could be exceeded. It also avoids unnecessary interventions into the process because these interventions worsen the process. [Taguchi 1994]

This article compares the most commonly used methods of statistical quality control (control charts - in our case the control chart of individual values) with the Taguchi's method of Partial Correction. The comparison applies to an actual process of hole reaming, and a test is carried out of gradual change of setting (continuously increasing simulation deviation).

\section{METHODS}

\subsection{Control charts}

A control chart is a graph that shows the state of process variability in time. Its aim is to give an indication about the state of action of definable causes. These indications must be reliable in terms of validity of intervention into the process to avoid unnecessary interventions, and must have the correct timing. For this control, the control charts generally comprise three basic lines: centre line $(\mathrm{CL})$, upper control limit $(\mathrm{UCL})$ and lower control limit (LCL). The space between UCL and $L C L$ is defined by the zone where only a random variability should be present. If e.g. one value is located above the UCL limit, the process is not statistically stable and it is necessary to intervene into the process and remove the causes, i.e. the definable cause is active in the process. These model situations can be multiple; therefore there are tests of non-random subgroups, providing the identification of situations which are not a natural component of the process. It should be added that each control chart has its own statistical conditions for use. [Jiju 2003]

This article employs the control chart of individual values (the control chart of Shewart type with the data evaluated by measurement). These control charts assume that the monitored factor can be described by a normal probability distribution. Another condition is a mutual statistical independence between the individual measurements of quality factor. In this type of control chart, it is absolutely essential to perform a verification of data normality. [Korzenowski 2015]

The control chart of individual values is used very often in the situation where automation allows the control; therefore the sorting into subgroups is not beneficial. In the control chart of individual values, two graphs are usually present; the former for the individual value $x i$, the latter for the moving range (MR).

Similarly to other control charts, the graphs consist of points plotted within the control limits, or natural tolerance limits of the process. The points outside these limits are the signals indicating that the process is not stable. Furthermore, other signals were specified; these indicate certain changes in the process (definable causes of fluctuations):

- Nine points in succession are located in the zone $\mathrm{C}$ or beyond

- Six points in succession are continuously increasing or decreasing

- Fourteen points in succession periodically fluctuate up and down

- Two of the three points in succession are in the zone A or beyond

- Four of the five points in succession are in the zone B or beyond

- Fifteen points in succession are in the zone $\mathrm{C}$ (above and below the central line)

- Eight points in succession are located at both sides of the central line, but none in the zone $C$

\subsection{Taguchi's Partial Correction technique}

Control charts are a very powerful tool for quality assurance of the product; however, they have their drawbacks. In practice, you can meet the approach when the process is not controlled 
on the basis of statistical investigation, but on the basis of empirical experience of employees. [Taguchi 1994] Savings on inspections will consequently translate into increased costs related to a poor quality production. [Aravidnan 1995]

Therefore, methods are sought that are a compromise between the quality of the product, ease of method of application and production costs. One of them is Taguchi's Partial Correction Technique (or Beta Correction Technique). This method provides measurement intervals and intervening limits in dependence on the costs associated with non-conforming products, and also on the costs of inspection during production. [Gijo 2014]

To effectively use the Partial Correction Method, it is necessary to comply with certain assumptions. These are as follows:

- Possibility of a relatively high frequency of measurement (especially when examining the process)

- Stable standard deviation must be available, i.e. the process must be in statistical control

- Measured characteristic must be quantitative

- Characteristic must be measurable

- Measuring system must be competent

- Short control cycle (the time between sampling, measurement, evaluation, and subsequent intervention into the process)

- Controlled process

Therefore, compared to SPC, after exceeding the limit, machining centre is not adjusted to the target value, but the size of correction is dependent on the distance from the target value of the process and the size of the standard deviation of the process concerned. [Taguchi 1994]

Relations for determining the coefficient $\beta$ are shown in Table 1.

\begin{tabular}{|c|c|c|}
\hline Condition & $\begin{array}{c}\text { Value of correction } \\
\text { coefficient }\end{array}$ \\
\hline$\beta \quad\left(x-x_{0}\right)^{2} \leq \sigma^{2}$ & 0 \\
\hline $\left.3-x_{0}\right)^{2} \geq \sigma^{2}$ & $1-\frac{\sigma^{2}}{\left(x-x_{0}\right)^{2}}$ \\
\hline
\end{tabular}

Table 1. Calculation of correction coefficient $\beta$

where $x_{0}$ - target value (nominal), $x$ - measured value, $\beta-$ correction coefficient.

Size of correction $(\Delta K)$ is then calculated according to the relation 1.

$$
\Delta K=-\beta \cdot\left(x-x_{0}\right)
$$

Uncertainty of estimate $\bar{X}$ is also compensated by a suitably designated interval of measurement.

\subsection{Case study-description}

The subject of measurement is a cylindrical hole having dimensions of $\varnothing 35+/-0.023$. Technologically, this dimension is ensured by drilling, roughing and subsequent reaming. Measured values after reaming are 200 pieces.

Technologically, this process is debugged, nevertheless occasionally definable causes occur; our team intends to eliminate them through interventions into the process adjustment. [Brezina 2012]

Given that the holes after drilling and roughing are not exactly circular and straight in the axial direction, they do not have the required dimensions within tolerance and desired roughness; then the last tools within the operations of CNC machining centre are reamers. [Holub 2014] Within this technology, reaming has allowance of about $0.3 \mathrm{~mm}$ for diameter of reaming. Reaming parameters are as follows: feed rate of $0.95 \mathrm{~mm} / \mathrm{rev}$, revolutions $n=50(1 / \mathrm{min})$, cutting speed $v=5.6 \mathrm{~m} / \mathrm{min}$, and in practice verified tool edge life $\mathrm{T}=60 \mathrm{~min}$. For our purpose, the eight-edged roller reamer is used. Mechanical reaming is followed by a final treatment by one-edged cemented carbide reamer, which guarantees the prescribed surface roughness Ra of 0.15. [Schützer 2014] The measured data are collected prior to this final operation.

For measuring of dimensions, a coordinate-measuring machine (CMM) is used. Because of higher temperature after machining and the necessary cooling of the part to $20^{\circ} \mathrm{C}$, there is a delay between production and measurement; for this reason, every $15^{\text {th }}$ piece is measured.

\subsection{The principle of comparing the methods of statistical process control}

To compare the above described methods, it is necessary to know the behaviour of the process over time; whether it is in statistical control, and how large the standard deviation is. Therefore, the comparison always starts from the analysis of the process. Data were analyzed with the Minitab software.

Measured data (200 values) obtained from the process of reaming were tested for normal distribution. It was found out that these data are normally distributed (see figure 1). $p$-Value of the normality test was 0.535 .

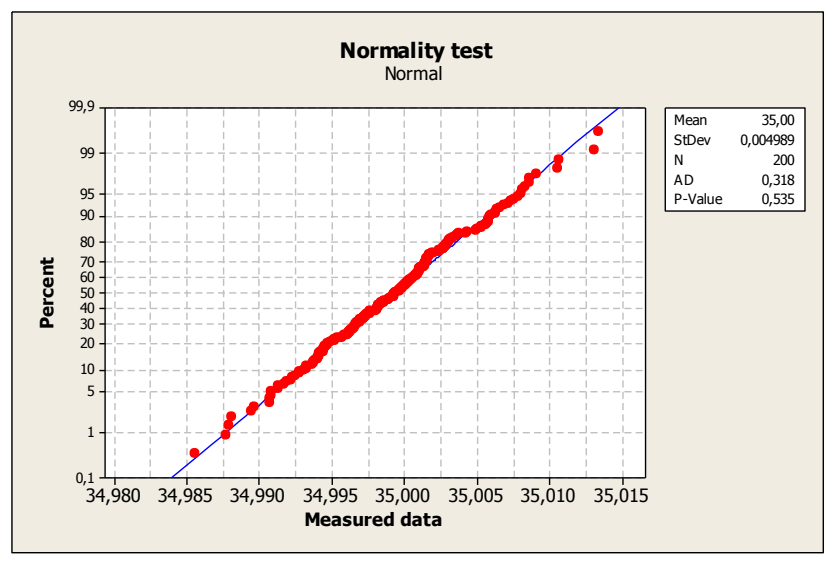

Figure 1. Results of normality test - Anderson Darling

Subsequently, a continuously growing simulation correction was introduced and added to the measured values. A size of correction of simulation is $0.0001 \mathrm{~mm}$ per one step. The measured data and the data corrected with simulation deviation are shown in the following figure.

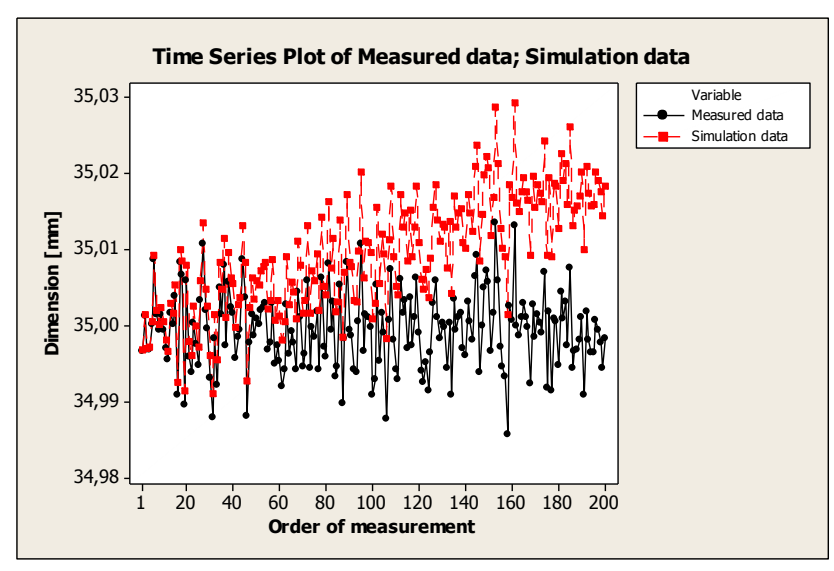

Figure 2. Measured data and the data corrected with simulation deviation $0.0001 \mathrm{~mm}$ per one step 


\section{RESULTS}

Data were analyzed in the control chart of individual values; immediately after the process had been evaluated as unstable, an automatic correction of the process was introduced (the corrected value was calculated as the difference between the so far measured average value and the target value).

The following graphs show a control charts of measured data and control charts of simulation data with marked points of corective actions.

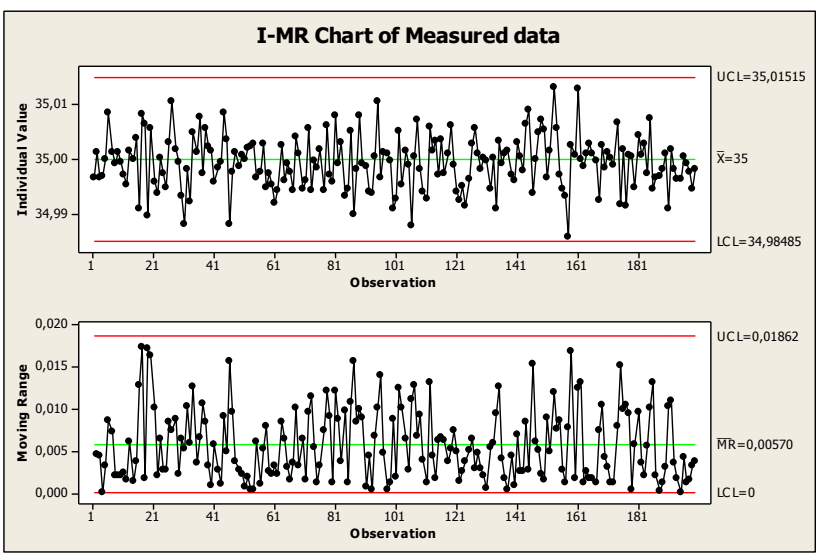

Figure 3. Control chart of measured data

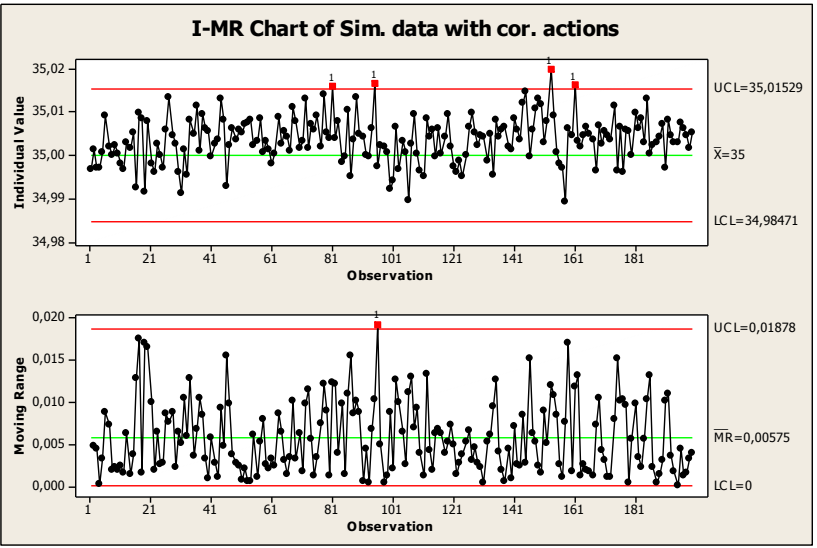

Figure 4. Control chart of corrected simulation data with marked points of corective actions

Furthermore, the data were analyzed using the Partial Correction Method. Each measured value was assigned the value of correction. The intervention into the process was simulated by adding the correction to all the values affected by the intervention. The following graph shows the control chart of data corrected with Partial Correction Method.

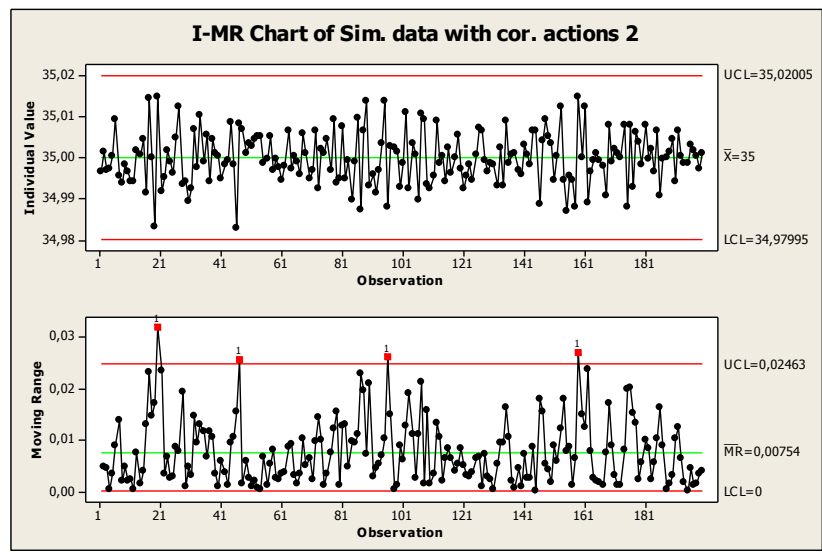

Figure 5. Control chart of Beta corrected simulation data with marked points of corective actions

The output of comparing of the test deviation with correction from Control chart and Partial correction method is shown in the figure below.

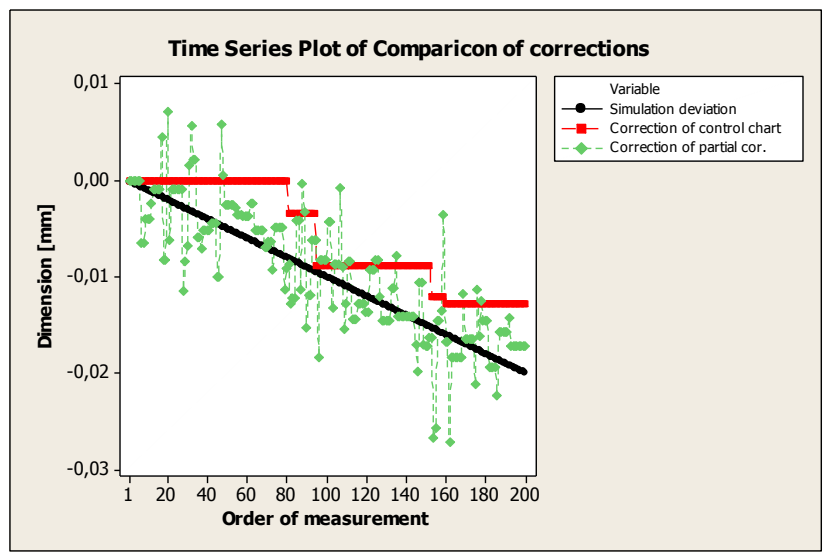

Figure 6. Comparison of test deviation with correction from Control chart and Partial correction

\section{DISCUSSION}

Our reaming workplace used a control chart of individual values and the Partial Correction Method. The aim of this article was to compare the results obtained using both approaches. The comparison showed that SPC implemented in this particular workplace maintained a certain quality threshold, but failed to provide a sufficient stability of the process.

The obtained findings show that the Partial Correction Method used for the studied process was able to provide a sufficient level of quality. The process became stabilized in position but the moving range increase. From the Figure 5 it is evident that there was a stabilisation of position.

In those cases where the degree of variation is very small, the stabilization of position is sufficient. In practice - accurate of machining is more accurate than sensitivity of measurement, the resolution of a small number of level values leads to extremely narrow limits; even excellent regulation process is apparently unstable.

In certain respects, the Partial Correction Method exceeded our expectations. In comparison with the application of a control chart at the workplace concerned, variability was reduced and the centering process was improved.

The advantages of Partial Correction Method include a simple implementation; there is no need for special training of operators. A disadvantage is that it does not provide so much information about the process as SPC does (e.g. trends are not 
seen - it is necessary to further evaluate the entered values). Moreover, this method has not been so far described in the way as control charts; there is a lack of practical experience.

\section{ACKNOWLEDGEMENT}

This work is an output of cooperation between internal BUT research project Reg. No. FSI-S-14-2401 and NETME Centre, regional $R \& D$ centre built with the financial support from the Operational Programme Research and Development for Innovations within the project NETME Centre (New Technologies for Mechanical Engineering), Reg. No. CZ.1.05/2.1.00/01.0002 and, in the follow-up sustainability stage, supported through NETME CENTRE PLUS (LO1202) by financial means from the Ministry of Education, Youth and Sports under the "National Sustainability Programme I".

\section{REFERENCES}

[Aravidnan 1995] Aravindan, P., et al. Continuous quality improvement through Taguchi's online quality control methods. International Journal of Operations \& Production Management, 1995, Vol.15, No. 7., pp. 60-77.

[Atienza 1997] Atienza, O.O., et al. Statistical process control and forecasting. International Journal of Quality Science, 1997, Vol.2, No. 1., pp. $37-51$.

[Brezina 2012] Brezina, T., at al. Simulation behavior of machine tool on the base of structural analysis in multi-body system. In: Proceedings of the 15th International Conference on Mechatronics, Mechatronika 2012, Prague, 5-7 December, 2012. Prague: Czech Technical University, pp 347-350.

[Gijo 2014] Gijo, E.V. and Scaria, J. Process improvement through Six Sigma with Beta correction: A case study of manufacturing company. International Journal of Advanced Manufacturing Technology, 2014, Vol. 71, No. 1-4., pp. 717-730.

[Holub 2014] Holub, M. and Knobloch, J., Geometric accuracy of CNC machine tools. In: Proceedings of the 16th International Conference on Mechatronics, Mechatronika 2014, Brno, 3-5 December, 2014. Brno: Institute of Electrical and Electronics Engineers Inc., pp 260-265.

[Jiju 2003] Jiju, A. and Tolga, T. A conceptual framework for the effective implementation of statistical process control. Business Process Management Journal, 2003, Vol. 9, No. 4., pp. 473-489. [Korzenowski 2015] Korzenowski, A. L., at al. Control charts for flexible and multi-variety production systems. Computers and Industrial Engineering, 2015, Vol. 88, pp. 284-292.

[Schützer 2014] Schützer, K., at al. Experimental investigation of hole quality during reaming applications using multi-blade tools. Journal of the Brazilian Society of Mechanical Sciences and Engineering, 2014, Vol. 36, No. 4., pp. 797-806.

[Taguchi 1994] Taguchi, G. Taguchi methods: On-line Production. New York: Amer. Supplier Inst., 1994.

\section{CONTACTS}

Ing. Lubos Kotek, Ph.D.

Institute of Production Machines, Systems and Robotics

Brno University of Technology, Faculty of Mechanical

Engineering

Technicka 2896/2, 61669 Brno, Czech Republic

tel.: +420 541142391 e-mail: kotek.l@fme.vutbr.cz

http://www.fme.vutbr.cz/ 